\title{
Distopías y la rebelión de las máquinas: Sobre los tópicos del Cyberpunk.
}

\author{
Dystopia and the Rebellion of the Machines: On the Topics of Cyberpunk.
}

\author{
Tadeo Masís González \\ Roberto Castro López ${ }^{2}$
}

Fecha de recepción: 24-2-20

Fecha de aceptación: 16-3-21

"De día, los bares de Ninsei estaban cerrados y no se distinguían unos de otros: el neón apagado, los hologramas inertes, esperando bajo el envenenado cielo de plata."

Neuromancer, William Gibson (2001)

\section{Resumen}

Se denomina cyberpunk a un segmento de la ciencia ficción desarrollado a partir de la década de los ochenta, cuya principal característica es su mirada crítica hacia la tecnología. El presente artículo tiene por objeto realizar una reflexión sociológica sobre este subgénero de la ciencia ficción, particularmente en torno a los temas presentes en este conjunto de obras y su relación con la modernidad. El análisis aborda especialmente las obras cinematográficas del cyberpunk en términos de cuatro tópicos: La noción de determinismo tecnológico en sus diferentes acepciones, el temor por parte de los seres humanos a no controlar las máquinas (la noción de rebelión de las máquinas), las distopías y el cyberpunk como crítica a la modernidad.

Palabras clave: Ciencia ficción, industria cultural, sociología de la tecnología, cultura científica, modernidad.

\begin{abstract}
Cyberpunk is known as a science fiction sub-genre that was developed during the eighties, whose main characteristic is its critical view on technology. The purpose of this article is to make a sociological analysis on this sub-genre of science fiction, particularly around the themes present in this set works and their relation with modernity. This analysis addresses the following four topics of cyberpunk: The notion of technological determinism in its different meanings, human beings fear not to control machines (the notion of machine rebellion), the dystopia and cyberpunk as a criticism of modernity.
\end{abstract}

Keywords: Science fiction, Cultural industry, Sociology of technology, Scientific culture, Modernity.

1 Bachiller en Administración Pública. Estudiante de sociología, Universidad de Costa Rica, Costa Rica. Correo electrónico: tadeo.masis@ ucr.ac.cr 


\section{Introducción}

El cyberpunk es un género literario y cinematográfico desarrollado en la última mitad del siglo XX, este género puede concebirse como una rama de la ciencia ficción. La particularidad del cyberpunk radica en su estética y sus tópicos, fundamentalmente caracterizados por incorporar elementos críticos en torno a la tecnología como los futuros distópicos o la noción de la rebelión de las máquinas. Según Gubern (2014):

[...] junto a la ciencia ficción celebrativa y circense coexistió un pequeño segmento de ciencia ficción especulativa y crítica, que obtuvo una de sus mejores formulaciones en Blade Runner (1982) en donde Ridley Scott adaptó una novela de Phillip K. Dick para presentar una opresiva ciudad de Los Ángeles, futurista e hipercontaminada”. (p.507)

Este subgénero de la ciencia ficción se inaugura en los años ochenta, siendo precisamente Blade Runner (1982) una de sus obras fundacionales, como también las novelas Neuromancer (1984) de William Gibson y Schismatrix (1985) de Bruce Sterling (traducida al español como Cismatrix en 2005). Dicha época se caracteriza por el cuestionamiento al proyecto de la modernidad, lo que necesariamente incide en las temáticas que abordan estas obras; como lo describen Múnera y Calderón (2014): "En la década de los 80 del siglo XX, un grupo de escritores de ciencia ficción crearon relatos en contextos distópicos y postindustriales de sociedades hipertecnologizadas, en contraposición de la visión utópica de la tecnología” (p.126).

Pese a su dimensión crítica, el cyberpunk no ha sido un género marginal en el mercado, este ha sido adoptado por las grandes industrias culturales, siendo el cine de Hollywood su principal vitrina para el consumo masivo. Además, está presente en la literatura, la música electrónica, la industria de los videojuegos y en gran cantidad de obras de animación japonesa (anime), cuyo principal exponente es la película Akira (1988) dirigida por Katsuhiro Otomo.

El presente artículo aborda algunos de los temas recurrentes del cyberpunk en términos de su relevancia social y filosófica, particularmente en relación con la modernidad. Dada la gran diversidad de materiales que pueden concebirse como parte del cyberpunk, este análisis hace énfasis en sus obras cinematográficas. En un primer apartado se plantea la manera en que las obras incorporan el determinismo tecnológico, propio del modelo tecnológico-científico de occidente. En un apartado posterior, se examina el temor a no controlar la máquina, usualmente mediado por la figura de la inteligencia artificial. Un tercer apartado aborda la noción de la distopía (o anti-utopía) presente en el cyberpunk. En un cuarto apartado se desarrolla una reflexión sobre el tipo de modernidad a la que alude este concepto y su relación con la noción de progreso. Finalmente, se plantea una conclusión en donde se discute la relevancia del cyberpunk como crítica a la tecnología, como antiutopía y como retrato de la hipermodernidad.

\section{Determinismo tecnológico}

Como se mencionó anteriormente, el cyberpunk es un subgénero de la ciencia ficción con elementos críticos en torno a la tecnología; una de las temáticas presentes en este conjunto de obras es aquello que Lander (1992) describe como el modelo científico-tecnológico de occidente, o lo que también podemos denominar como determinismo tecnológico:

[...] la lógica de este modelo implica que el desarrollo societal es un proceso de reducción de la subjetividad humana por el cálculo racional. El control sobre los hombres y las cosas es asegurado mediante la sustitución de la interpretación humana por la racionalidad tecnológica en la organización de cualquier actividad. La subjetividad se subordina a la objetividad. Una importante consecuencia de este desarrollo es que la Tecnología tiende a moldear a quien la usa, y no sólo en las formas sugeridas por el materialismo cultural, específicamente, la tecnología moldea quien la usa alterando los paradigmas de la sociedad, esto es, reemplazando relaciones sociales por nexos determinados tecnológicamente. (p.10) 
De esta manera, el determinismo tecnológico es la noción de que la tecnología es una fuerza impersonal que determina los cambios en la sociedad. Es decir, bajo esta óptica los seres humanos estarían subordinados al desarrollo tecnológico, ya sea en términos de las propias innovaciones tecnológicas o en términos de la lógica interna de las ciencias; por tanto, impera la idea de que científicos y técnicos desarrollan innovaciones con independencia de cualquier tipo de relación social. Aún y cuando se puede establecer una diferencia entre determinismo tecnológico fuerte y determinismo tecnológico suave (Chandler, 1995), la adscripción a estas ideas implica que la tecnología sea siempre interpretada como la variable independiente que explica los cambios en la cultura, la comunicación y el conjunto de la sociedad.

En obras como The Matrix (1999) de las hermanas Wachowski o The Terminator (1984) de James Cameron, es posible observar un trasfondo en que el desarrollo de tecnologías como robots o inteligencia artificial, suponen cambios profundos en las civilizaciones humanas. En otras palabras, la introducción de determinadas tecnologías y artefactos como androides o programas informáticos, necesariamente modifica el orden social, llevándolo en estos casos prácticamente a un colapso. Así, estas obras representan sociedades e individuos subordinados a la lógica del desarrollo tecnológico.

En cuanto a la noción de determinismo tecnológico, Gómez (1997) plantea que existen tres variantes de esta. En primer lugar, un determinismo nomológico, en el que el orden de los cambios tecnológicos determina completamente al orden de los cambios sociales. En segundo lugar, un determinismo normativo que supone cómo la tecnología es autónoma en tanto las normas que la rigen están al margen de la ética y la política. Por último, un determinismo de consecuencias involuntarias, en donde la tecnología es concebida como autónoma en tanto sus resultados y consecuencias son ajenas al control y la previsión de los seres humanos.

En el conjunto de las obras del cyberpunk se encuentran ejemplos de los distintos tipos de determinismo, sin embargo, se considera que el determinismo de consecuencias involuntarias está particularmente presente en el cyberpunk. Por ejemplo, en obras como Robocop (1987), Upgrade (2018), Ghost in the Shell (1995) y Blade Runner (1982), es posible observar la manera en que la tecnología se comporta de una manera ajena a la previsión de los seres humanos. De esta forma, la tecnología cobra un carácter autónomo, lo que usualmente altera (en menor o mayor medida) el funcionamiento de las sociedades retratadas en estas obras.

\section{Temor a no controlar la máquina.}

Otro tema recurrente en las obras del cyberpunk gira en torno a las máquinas, particularmente al temor de que los seres humanos no sean capaces de controlarlas. En términos generales, una máquina está formada por un complejo de agentes no orgánicos cuyo fin es cumplir una función para el ser humano (Mumford, 1992). Esto supone que en última instancia, una máquina sea un producto diseñado por el ser humano. Por dicha razón, la posibilidad de que las personas no controlen a las máquinas es un terreno especulativo, especialmente abordado en la ciencia ficción.

Para abarcar este tópico es pertinente considerar algunos elementos sobre el impacto epistemológico de la tecnología. El filósofo italiano Evandro Agazzi (1998) plantea que el uso de tecnología es una condición inherente al desarrollo de la ciencia moderna y a la producción de conocimiento. En este marco, lejos de haber dominado el mundo natural, el ser humano ha creado el mundo de las máquinas, de manera que: "la máquina no es algo que subyugue a la naturaleza sino que la sustituye” (p.28).

Dicha sustitución de la naturaleza por la máquina ocurre en la medida en que los seres humanos tienen la capacidad de controlar a las máquinas; mientras que la naturaleza mantiene su carácter autónomo e imprevisible. Es de esta forma que, según Agazzi, las máquinas se convierten en la herramienta necesaria para hacer ciencia empírica, puesto que estas van a permitir la experimentación y la previsibilidad en el quehacer científico. Por consiguiente, las máquinas carecen de la cualidad de ser impredecibles y autónomas en términos de su diseño y funcionalidad, debido a que las máquinas realizan únicamente lo que su diseño permite: siguiendo a Agazzi (1998): 
La máquina posee una enorme ventaja: dentro de ella no hay secretos, todo es conocido, quizás no para mí pero sí para el técnico o el especialista. Si se estropea, se puede arreglar, pues está construida según un proyecto que permite saber cómo funciona y devolverla a su funcionamiento. Antes de construirla sabemos cómo funcionará y por qué funcionará así y esto no se conoce por la experiencia propiamente dicha, sino que se sabe antes de que exista. (p.29)

En gran parte de las obras del cyberpunk se exploran escenarios especulativos en donde los seres humanos no controlan a la totalidad de las máquinas, situación en la que estas son usualmente representadas como robots o computadoras guiadas por inteligencia artificial que ganan autonomía frente a las personas. A su vez, dicho evento supone la representación de una sociedad en la que las máquinas llegan a poseer elementos desconocidos para los seres humanos, aún y cuando estos son originalmente sus creadores.

En los filmes anteriormente mencionados The Matrix (1999) y The Terminator (1984), es posible observar la noción de rebelión de las máquinas. En ambas obras, la inteligencia artificial desarrollada por los seres humanos (con el fin de automatizar determinadas labores), termina por sublevarse contra sus creadores y esclavizar a una parte de estos. En el caso de dichas obras, el impulso de las máquinas para rebelarse procede de un diagnóstico propio, mediante el cual determinan que los seres humanos representan una amenaza para su supervivencia.

En obras más recientes como Ex Machina (2014) y Upgrade (2018), se representan máquinas que se rebelan contra sus creadores inmediatos con el fin de ganar autonomía y vivir entre los humanos por su cuenta. En este caso, las máquinas desarrollan la capacidad de engañar y atacar (en función de sus propios intereses) a los científicos encargados de su diseño y producción, más allá de las previsiones humanas sobre las funciones de estas máquinas.
Todas estas representaciones de las máquinas como entidades con la capacidad de engañar y subyugar a los seres humanos, además de representar un tipo de relación entre ser humano y máquina, aluden a un tipo particular de hombre. En los distintos filmes mencionados en esta sección, los humanos son concebidos por las máquinas como fuentes de energía o como un medio para su liberación. La posibilidad de estos primeros sean instrumentalizados por las máquinas, apunta a una concepción de la humanidad "en simetría" con las máquinas y las cosas:

[...] si el hombre ha de ser concebido como una máquina o como una de las muchas cosas del mundo, no existe ninguna razón clara para rechazar servirse de él y tratarlo como se haría con una máquina u otros objetos naturales. (Agazzi, 200o, p.6o)

Así, una rebelión de las máquinas, además de llevar a la máquina al orden de lo ingobernable, implica posicionar a la humanidad al mismo nivel de las máquinas (o las "cosas"). En estas obras, el carácter imprevisible de la tecnología se complementa con la posibilidad de que estas utilicen al ser humano para la consecución de determinados fines. De esta forma, la pérdida de control sobre la inteligencia artificial representa también una "reificación” del hombre, una degradación al estatus de "cosa” o máquina.

Esta incapacidad del ser humano para controlar la tecnología representada en el cyberpunk, deriva usualmente en un enfrentamiento entre seres humanos y robots, lo cual, implica necesariamente una lucha del ser humano contra su propia obra tecnológica. De esta manera, el temor a no controlar la máquina se desagrega en un temor a perder control sobre el mundo de lo previsible, pero también en un temor a una nueva fuerza impredecible (además de la naturaleza) con capacidad de degradar lo humano al nivel de la máquina. 


\section{Distopías, el no futuro.}

Una de las características del cyberpunk es la representación de futuros distópicos, o lo que también se denomina como anti-utopías. Según Carvajal (2001), el propio término de Cyberpunk3 evoca un escenario de alta tecnología y bajo nivel de vida. Siguiendo a Carvajal, la particularidad de este movimiento estético radica en lo siguiente: "Este subgénero profundiza la visión pesimista de la tecnología. La crítica se orienta a la deshumanización provocada por el "imperativo tecnológico", es decir, realizar todo aquello que resulta técnicamente posible sin tomar en cuenta consideraciones éticas de ningún tipo" (s.p).

Dos obras que ilustran este uso indiscriminado de la tecnología son Johnny Mnemonic (1994) de Robert Longo y Metropolis (2001) de Shigeyuki Hayashi. La primera muestra un futuro en donde el inmenso flujo de información digital que emplea la humanidad, genera una enfermedad mortal para los mismos seres humanos; la segunda ilustra un futuro industrial y robotizado, en donde el imperativo por desarrollar un robot humanoide de grandes cualidades, pone en peligro a prácticamente toda la humanidad.

De esta forma, el cyberpunk representa futuros distópicos provocados por las consecuencias de un imperativo tecnológico. Por esta razón, el futuro descrito en estas obras sugiere sociedades hipertecnologizadas con escenarios de contaminación, desigualdad, exclusión y explotación mediados por la tecnología, como ocurre por ejemplo en la obra literaria Neuromancer (1984) y en filmes como Akira (1988), Total Recall (1990), Repo Men (2010), entre otros. En este marco, el cyberpunk se adscribe al pesimismo tecnológico; las representaciones del futuro que proyectan estas obras desde la década de los 8o, manifiestan un cuestionamiento a la noción tradicional de progreso originada en el marco de los desarrollos tecnológicos y científicos de la ilustración. En torno a este tipo de contenidos, Vizcarra (2013) plantea lo siguiente:
[...] hasta ahora los contenidos del cyberpunk no han alcanzado a proponer una imagen alternativa del futuro más allá de los finales felices. Parece que el agotamiento de las utopías es uno de los rasgos más notables de la miseria cultural del presente. (p.55)

Cabe añadir que en estas obras, las distopías cuentan usualmente con fuertes alusiones al corporativismo; los futuros distópicos se representan en muchos casos como sociedades capitalistas donde el mercado es el actor predominante en la sociedad, particularmente por medio de grandes corporaciones que desarrollan las últimas tecnologías donde incluso asumen funciones tales como la seguridad pública, como en el caso de RoboCop (1987) y la exploración espacial en el caso de Gattaca (1997).

Es pertinente relacionar las ideas presentes en estas producciones con la noción de realismo capitalista, Fisher (2017) la describe como: "la idea muy difundida de que el capitalismo no solo es el único sistema económico viable, sino que es imposible incluso imaginarle una alternativa." (p.22). De esta manera, el cyberpunk alude con frecuencia al realismo capitalista; aún y cuando las obras fundacionales de este subgénero se desarrollan en el contexto de la guerra fría, estas tienden a proyectar el futuro como una sociedad necesariamente capitalista.

Una manifestación representativa del realismo capitalista es la premisa del filme A.I. Artificial Intelligence (2001) dirigida por Steven Spielberg. En ella, los seres humanos situados en un futuro cercano han sufrido una catástrofe ambiental producto del derretimiento de los polos, lo que limita severamente las zonas habitables del planeta; la solución a esta problemática es el desarrollo de robots humanoides que solventan el faltante de humanos. Sin embargo, estos robots solo se conciben como producto de innovación empresarial y de este modo, se representa un mundo con grandes alteraciones geográficas y demográficas, pero con un sistema económico más o menos intacto.

3 Además de aludir a un bajo nivel de vida, el punk puede entenderse como antecedente del cyberpunk en tanto constituye una estética destructiva y veloz. Greil Marcus (1993) considera al género punk como el rechazo total a la música de la época así como el rechazo a la tradición. En ese sentido, el punk reniega de aquello que alguna vez fue exitoso o utópico. 


\section{Crítica a la modernidad}

El agotamiento de las utopías retratado en el cyberpunk ha sido interpretado como una crítica a la categoría de modernidad. En este marco se desprendería una reflexión sobre el fracaso del proyecto moderno: "El pensamiento crítico forjado en el contexto de la modernidad ha dado libertades, pero también a través de éste se ha vislumbrado el vacío. La modernidad no aporta elementos sólidos para la justificación de la existencia" (Vizcarra, 2013, p.55). Sin embargo, el cyberpunk no refleja necesariamente un rechazo total a la noción de futuro y a las promesas de la modernidad, sino que retrata las ideas subyacentes de una nueva modernidad, como la constituye la noción de hipermodernidad concebida por Lipovetsky (2018).

La modernidad inicial según Lipovetsky (2018), promulgaba una noción de progreso dotada de una connotación ideológico-política, orientada a grandes utopías colectivas; mientras que en la hipermodernidad, la noción de progreso se reorienta hacia una dimensión primordialmente tecno-científica donde predomina la figura del individuo frente a los grandes proyectos colectivos. En otras palabras: "el culto a la modernización técnica ha superado a la glorificación de los fines y los ideales" (p.6o).

En este marco, dado que tiende a proyectar futuros distópicos e hipertecnologizados, el cyberpunk remite a la noción de hipermodernidad; visto de otra manera, el cyberpunk refleja una ruptura con los grandes proyectos ideológico-políticos de la modernidad. No obstante, esto no implica una ruptura total con la idea de progreso ni tampoco una ruptura con la preocupación por el devenir de las sociedades, más bien alude precisamente a una de las características que Lipovetsky (2018) atribuye a la hipermodernidad:

Las utopías colectivas mueren, las actitudes pragmáticas de previsión y prevención técnicocientíficas se intensifican. Si bien el eje del presente es dominante, no es absoluto, ya que la cultura de prevención y «la ética del futuro» hacen que reaparezcan los imperativos de un después más o menos lejano. (p.72)
El cyberpunk manifiesta una cultura de prevención y una ética del futuro cuando plantea escenarios del porvenir en sociedades contaminadas, segregadas y donde prima el uso indiscriminado de la ciencia y la tecnología. Esto refleja una preocupación legítima por dicho futuro, pero en un clima de incertidumbre, despojado de una confianza estable en el progreso tal y como también Lipovetsky (2018) caracteriza la modernidad actual: "La hipermodernidad no ha reemplazado la fe en el progreso por la desesperación y el nihilismo, sino por una confianza inestable, fluctuante, variable según los acontecimientos y las circunstancias" (p.74).

Es pertinente añadir que aún y cuando el cyberpunk se caracteriza por su visión crítica de la tecnología y puede considerarse afín al pesimismo tecnológico, también confiere a la inteligencia artificial la posibilidad de mejorar o prolongar la vida de los seres humanos en determinadas situaciones; por ejemplo, mediante procedimientos de la medicina avanzada o mediante la automatización de labores manuales como cocinar, conducir vehículos, trabajos pesados, entre otras. Es así como el cyberpunk critica la validez del proyecto de la modernidad, pero no implica una renuncia a los valores de dicha modernidad en su dimensión tecno-científica.

\section{Conclusión}

A través de sus obras cinematográficas, el cyberpunk refleja un conjunto de representaciones particulares sobre la relación entre tecnología y sociedad. Un primer tópico presente en estas obras es el determinismo tecnológico, principalmente aquel que concibe a la tecnología como autónoma y cuyas consecuencias son siempre incontrolables e imprevisibles. Esto es muestra de que el cyberpunk retrata el modelo científico-tecnológico de occidente, en donde la tecnología es aquella fuerza que determina en última instancia el cambio social.

Por otra parte, el carácter imprevisible de los efectos de la tecnología es un tema particularmente explorado en el cyberpunk, ya sea en términos técnicos como máquina o en términos del impacto ético y político de la tecnología en el devenir de las sociedades. En cuanto a las máquinas, por medio de la representación de la 
rebelión de estas, el cyberpunk refleja el temor humano a no controlar aquello que ha creado para satisfacer sus propias necesidades. Esto también supone un temor humano a perder previsibilidad sobre su obra tecnológica, así como a encontrar en las máquinas una nueva fuerza autónoma e incontrolable, asimilable a la naturaleza. La imprevisibilidad en términos del devenir de las sociedades se manifiesta en la proyección de futuros distópicos e hipertecnologizados.

La tendencia en estas obras a representar el futuro como una anti-utopía puede interpretarse como una crítica al proyecto original de la modernidad y la noción de progreso. Aunado a ello, el cyberpunk integra elementos de lo que se ha denominado como realismo capitalista. De esta manera, es posible plantear que la imposibilidad para imaginar futuros no distópicos está mediada por las relaciones de poder en el marco de un modo de producción capitalista y lo que implica en torno al acceso segregado a la producción y uso de tecnologías.

Finalmente, el cyberpunk lejos de constituir una crítica negacionista de la modernidad, representa muchas características de la hipermodernidad, en dónde las grandes utopías colectivas cargadas de valores ideológicopolíticos son reemplazadas por futuros individualizados e inciertos, pero orientados por la noción de progreso en su dimensión tecno-científica.

\section{Referencias bibliográficas}

Agazzi, E. (1998). El impacto epistemológico de la tecnología. Argumentos de Razón Técnica,1, pp.17-31.

Agazzi, E. (2000). Filosofía de la naturaleza. Ciencia y cosmología. México: Fondo de cultura económica.

Carvajal, A. (2001). El Cyberpunk: crítica a la tecnología informática. Revista Comunicación, 11 (4), (s.p).

Chandler, D. (1995). Technological or Media Determinism. Recuperado de http://visual-memory.co.uk/ daniel/Documents/tecdet/tdet11.html

Fisher, M. (2017). Realismo capitalista. ¿No hay alternativa?. Buenos Aires: Caja Negra Editora.

Gibson W. (1984). Neuromancer. New York: Ace Books.

Gibson, W. (2001). Neuromante. Barcelona: Ediciones Minotauro.

Gómez, R, J. (1997). Progreso, determinismo y pesimismo tecnológico. Redes, 4(10), 59-94.

Gubern, R. (2014). Historia del cine. Barcelona: Editorial Anagrama.

Lander E. (1992). La ciencia y la tecnología como asuntos políticos. Límites de la democracia en la sociedad tecnológica. Caracas: Editorial Nueva Sociedad.

Lipovetsky, G. (2018). Los tiempos hipermodernos. Barcelona: Editorial Anagrama.

Marcus, G. (1993). Rostros de carmín. Una historia secreta del siglo XX. Barcelona: Editorial Anagrama.

Mumford, L. (1992). Técnica y civilización. Madrid: Alianza Editorial. 
Múnera, C., y Calderón, D. (2014). La televisión del control neuronal y la teoría moral de los muñecos de trapo: sentidos del cyberpunk y postcyberpunk en el cine, e impacto de la tecnología en la sociedad. Revist Temas, 3(8), pp.125-137.

Sterling, B. (2005). Cismatrix. Madrid: Bibliópolis.

Vizcarra, F. (2013). La mirada cómplice. Ensayos sobre cine y sociedad. México: CONACULTA.

\section{Obras cinematográficas:}

Cameron, J. (Director). (1984). The Terminator [Filme]. Hemdale Film Corporation.

Garland, A. (Director). (2014). Ex Machina [Filme]. Film4 Productions.

Longo, R. (Director). (1995). Johnny Mnemonic [Filme]. Alliance Communications.

Niccol, A. (Director). (1997). Gattaca [Filme]. Columbia Pictures.

Oshii, M. (Director). (1995). Ghost in the Shell [Filme]. Kodansha.

Otomo, K. (Director). (1988). Akira [Filme]. Tokyo Movie Shinsha.

Sapochnik, M. (Director). (2010). Repo Men [Filme]. Relativity Media.

Scott, R. (Director). (1982). Blade Runner [Filme]. Warner Brothers.

Shigeyuki, H. (Director). (2001). Metropolis [Filme]. Madhouse.

Spielberg, S. (Director). (2001). A.I Artificial Inteligence [Filme]. DreamWorks.
Verhoeven, P. (Director). (1987). RoboCop [Filme]. Orion Pictures Corporation.

Verhoeven, P. (Director). (1990). Total Recall [Filme]. Carolco Pictures.

Wachowski, L., y Wachowski, L (Directoras). (1999). The Matrix [Filme]. Village Road Pictures.

Whannell, L. (Director). (2018). Upgrade [Filme]. Blumhouse Productions. 